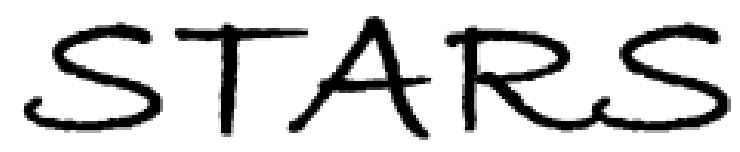

University of Central Florida

STARS

$1-1-2006$

\title{
External cavity multiwavelength semiconductor hybrid mode- locked laser intracavity gain dynamics
}

Luis C. Archundia

University of Central Florida

Peter J. Delfyett Jr.

University of Central Florida

Find similar works at: https://stars.library.ucf.edu/facultybib2000

University of Central Florida Libraries http://library.ucf.edu

This Article is brought to you for free and open access by the Faculty Bibliography at STARS. It has been accepted for inclusion in Faculty Bibliography 2000 s by an authorized administrator of STARS. For more information, please contact STARS@ucf.edu.

\section{Recommended Citation}

Archundia, Luis C. and Delfyett, Peter J. Jr., "External cavity multiwavelength semiconductor hybrid modelocked laser intracavity gain dynamics" (2006). Faculty Bibliography 2000s. 5912.

https://stars.library.ucf.edu/facultybib2000/5912

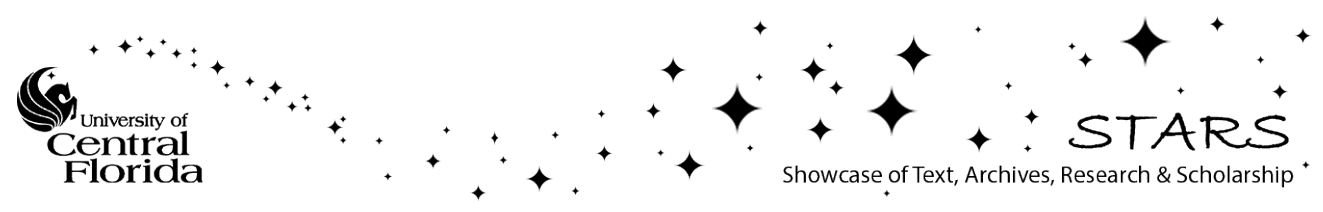




\section{External cavity multiwavelength semiconductor hybrid mode-locked laser intracavity gain dynamics}

Cite as: Appl. Phys. Lett. 88, 191117 (2006); https://doi.org/10.1063/1.2203228

Submitted: 10 January 2006. Accepted: 28 March 2006. Published Online: 11 May 2006

Luis C. Archundia, and Peter J. Delfyett

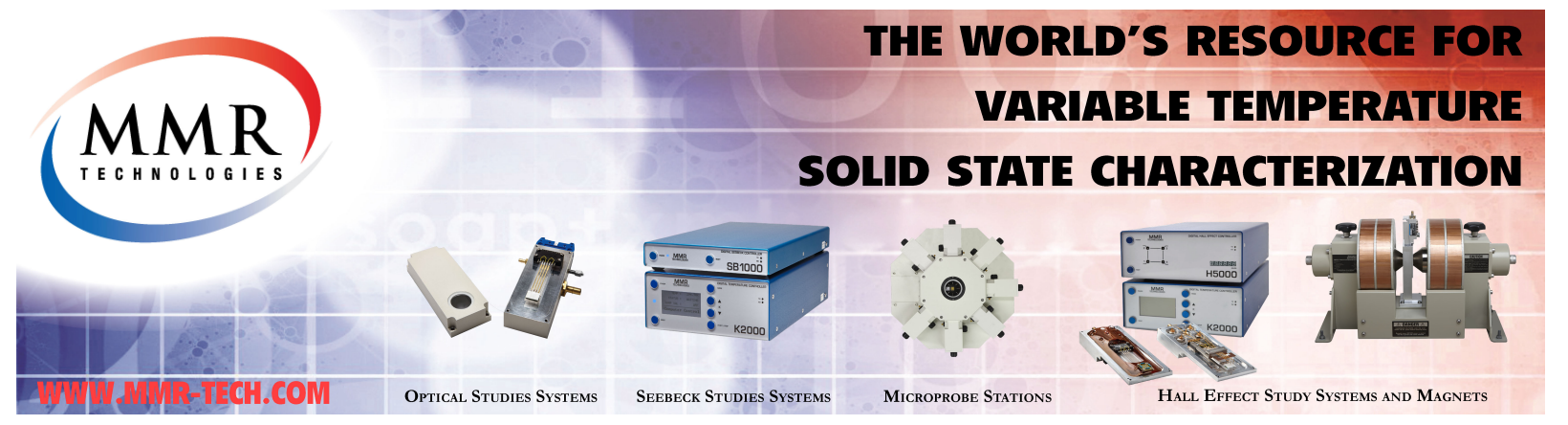




\title{
External cavity multiwavelength semiconductor hybrid mode-locked laser intracavity gain dynamics
}

\author{
Luis C. Archundia ${ }^{a)}$ and Peter J. Delfyett, Jr. \\ CREOL and FPCE / College of Optics and Photonics, University of Central Florida, \\ 4000 Central Florida Boulevard, Orlando, Florida 32816
}

(Received 10 January 2006; accepted 28 March 2006; published online 11 May 2006; publisher error corrected 16 May 2006)

\begin{abstract}
The intracavity gain dynamics of an external cavity semiconductor hybrid mode-locked laser is measured under multiwavelength operation. The results show a temporal skew between pulses corresponding to different wavelength channels. A measurement of the temporal evolution of the gain reveals a slow gain depletion, avoiding carrier heating and carrier cooling, and decreasing self-phase modulation effects and gain competition between wavelength channels, making multiwavelength operation possible. (C) 2006 American Institute of Physics.
\end{abstract}

[DOI: $10.1063 / 1.2203228]$

Wavelength division multiplexing (WDM) is an important tool used to increase the information carrying capacity of optical fiber networks; ${ }^{1}$ hence the need for reliable and inexpensive multiwavelength sources is evident. Multiwavelength semiconductor lasers have proven to be attractive candidates for both WDM and ultrawideband photonic signal processing applications. $^{2-5}$ In order to understand the mechanisms that support multiwavelength operation of semiconductor mode-locked lasers, we report in this article the measurements of the intracavity gain dynamics of an external cavity multiwavelength hybrid semiconductor mode-locked laser. The results show a slow depletion of the gain, resulting in a reduction of the integrated self-phase modulation and the carrier heating and carrier cooling effects observed in semiconductor optical amplifiers (SOAs) for the amplification of short pulses. ${ }^{6,7}$ The reduction of the nonlinearities and a transient unsaturated gain enable the multiwavelength operation of the laser. In addition, it was observed that pulses corresponding to different wavelength channels do not coincide temporally and are partially correlated.

The laser under investigation is sketched in Fig. 1, and is an external cavity hybrid semiconductor mode-locked laser in a multiwavelength configuration. The gain media is a multiple quantum well AlGaAs semiconductor optical amplifier. The SOA is $500 \mu \mathrm{m}$ long and is antireflection coated with the gain stripe tilted $6^{\circ}$ with respect to the facets to prevent feedback due to the residual facet reflectivity. ${ }^{8}$ The laser output is obtained by using a $50 \%$ output coupler. A multiple quantum well saturable absorber is used as a passive modelocking element, ${ }^{9}$ and an etalon in combination with a spectral filter is used to establish multiwavelength operation. The etalon is a $0.5 \mathrm{~mm}$ thick solid glass with $70 \%$ reflecting mirrors resulting in transmission peaks separated $0.48 \mathrm{~nm}$ at $835 \mathrm{~nm}$, with a finesse of 8 . The spectral filter consists of a 1800 lines/mm grating, a $15 \mathrm{~cm}$ focal length lens, a mirror, and a slit. The grating, lens, and mirror are located $15 \mathrm{~cm}$ from each other (lens' focal length) and the slit is used to select the desired spectral bandwidth. In this case, the spectral filter is configured to allow the amplification of three

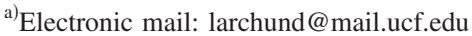

etalon transmission peaks around $835 \mathrm{~nm}$ (three modelocked wavelength channels).

Short probe pulses are necessary to measure the gain dynamics of the multiwavelength laser. An external cavity hybrid semiconductor mode-locked laser in a linear configuration $^{7}$ and a grating pulse compressor ${ }^{10}$ were used as a source of probe pulses, generating pulses with a temporal duration of approximately $1 \mathrm{ps}$, with a center wavelength of $839.5 \mathrm{~nm}$ and $3 \mathrm{~nm}$ spectral bandwidth. The multiwavelength laser and the probe laser are synchronized by having the same cavity length and sharing the same radio frequency source. To measure the relative timing jitter between both lasers, the cross correlation of pulses from the multiwavelength laser with pulses from the probe laser was measured. For this measurement, the multiwavelength laser was temporarily configured to obtain short pulses by removing the etalon and adjusting the spectral filter to allow a broader spectral bandwidth, obtaining pulses of approximately 1 ps after dispersion compensation. The pulse to pulse timing jitter is equal to the broadening of the cross correlation over the theoretical width of the cross correlation calculated from the autocorrelation width of each pulse. ${ }^{11}$ The measured timing jitter using this technique is $2 \mathrm{ps}$. To corroborate this result, a fast photodetector was used to detect the pulses from the multiwavelength laser, and this photodetector signal was used to trigger a sampling scope measuring the probe laser pulses, measuring a relative timing jitter of approximately 2 ps between the two lasers.

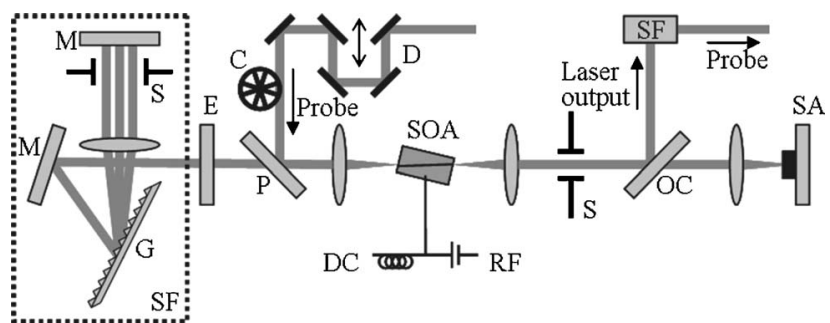

FIG. 1. External cavity multiwavelength semiconductor hybrid modelocked laser. M, mirror, S, slit, G, grating, SF, spectral filter, E, etalon, C, chopper, P, pellicle beam splitter; D, variable delay, SOA, semiconductor optical amplifier; DC, direct current; RF, radio frequency; OC, output coupler; and SA, saturable absorber. 

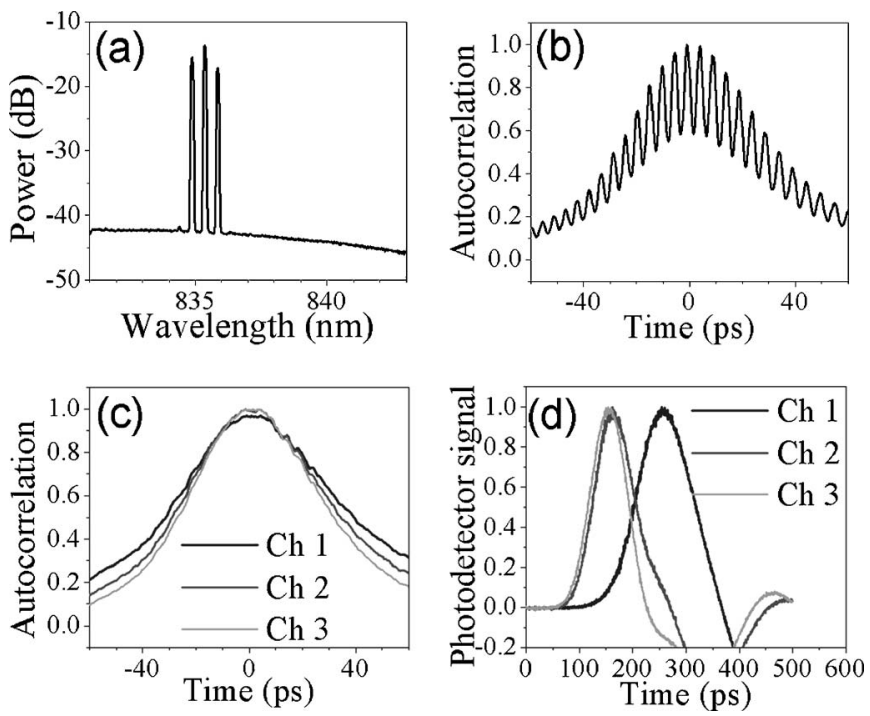

FIG. 2. (a) Multiwavelength pulse spectrum, (b) multiwavelength pulse autocorrelation, (c) autocorrelation of each individual wavelength channel, and (d) relative time pulse position for each individual wavelength channel.

After the timing jitter measurement, the multiwavelength laser was set back to the three wavelength configuration. Hybrid mode-locking was achieved for a SOA direct current bias of $150 \mathrm{~mA}$ with $250 \mathrm{~mW}$ of radio frequency signal power at $450 \mathrm{MHz}$, yielding $4 \mathrm{~mW}$ of average output power. By adjusting the laser cavity, it is possible to obtain wavelength channels with approximately the same spectral peak intensity. An identical SOA was used as a power amplifier to amplify the multiwavelength pulses to approximately $20 \mathrm{~mW}$. The multiwavelength pulse spectrum is shown in Fig. 2(a), and the autocorrelation is shown in Fig. 2(b). Due to the relatively long pulse duration and the limited time window of the autocorrelator used, a good estimate of the pulse duration of the multiwavelength pulses cannot be obtained. The modulation observed in the autocorrelation of the multiwavelength pulses indicates a partial correlation between wavelength channels.

The individual wavelength channels are separated by using a spectral filter similar to the filter in the multiwavelength laser cavity. The autocorrelation of each individual wavelength channel is measured and the results are plotted in Fig. 2(c). The relative temporal pulse position for each wavelength channel is measured using a $15 \mathrm{GHz}$ fast photodetector and a sampling scope and it is shown in Fig. 2(d). Note that the negative signal values in Fig. 2(d) are an artifact from the impulse response of the photodetector. Figure 2(d) shows that the pulses do not temporally coincide. The SOA gain and the laser dispersion determine the intensity and the relative temporal pulse position of each channel, and external cavity semiconductor mode-locked lasers work in a regime where there is a transient unsaturated gain, which decreases gain competition among wavelength channels.

To measure the temporal evolution of the SOA gain in the multiwavelength laser, the probe pulses are introduced into the laser cavity by using a pellicle beam splitter as shown in Fig. 1. The probe power introduced (a few microwatts of average power) is sufficiently low to avoid disturbing the operation of the laser. Prior to introducing the probe pulses into the multiwavelength laser, a variable delay is used to change the relative delay between the probe and the multiwavelength pulses in the multiwavelength laser cavity.
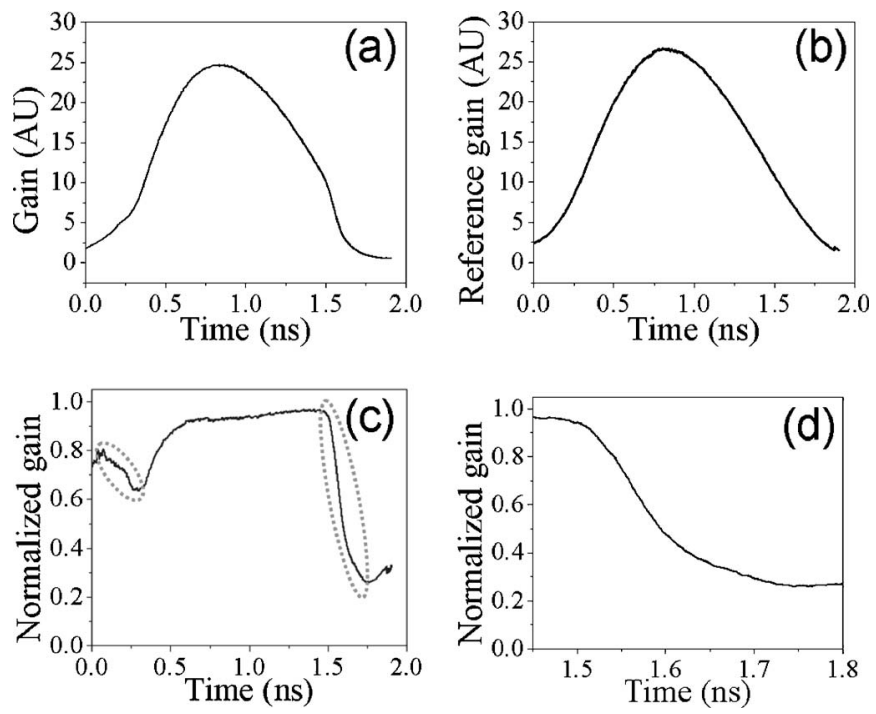

FIG. 3. (a) Temporal evolution of the SOA gain, (b) gain modulated by the bias current, (c) normalized gain (the gain depletions due to the amplification of the multiwavelength pulses are circled), and (d) gain depletion close-up.

The probe pulses measure the SOA gain as a function of the delay between the probe and the multiwavelength pulses, which represents the temporal evolution of the SOA gain.

The laser output contains the multiwavelength pulses and the probe pulses after measuring the SOA gain; however, the probe and the multiwavelength laser spectra do not overlap, facilitating their separation via spectral filtering. To obtain clean measurements, a chopper and a lock-in amplifier are used on the probe beam. Figure 3(a) shows the measured temporal evolution of the SOA gain. The gain depletion and gain recovery signal associated with the amplification of the multiwavelength pulses is superimposed on a gain signal modulated by the bias current. This modulated gain is obtained by measuring the temporal evolution of the gain while blocking the laser feedback, and is used as a reference to normalize the measured gain under multiwavelength operation. The modulated gain is shown in Fig. 3(b) and the normalized gain is shown in Fig. 3(c). The chosen time window in Figs. 3(a)-3(c) is sufficient to observe the salient gain features during one cavity round trip. Times beyond this window correspond to a slow gain recovery between the amplification of consecutive pulses.

Due to the multiwavelength laser cavity configuration, the multiwavelength pulses pass throughout the SOA two times per round trip, traveling in opposite directions each time, while the probe pulses travel in only one direction. Two gain depletions can be observed in Fig. 3(c). The first corresponds to the multiwavelength and the probe pulses traveling in opposite directions in the SOA and the second corresponds to multiwavelength and probe pulses traveling in the same direction in the SOA. A close-up of the second gain depletion is shown in Fig. 3(d). The important feature is that the SOA gain depletes slowly, thus avoiding nonlinearities in the SOA during the amplification process.

In conclusion, the intracavity gain dynamics of an external cavity hybrid semiconductor mode-locked laser was measured under multiwavelength operation. It was shown how the pulses corresponding to different wavelength channels are temporally skewed, broadening the temporal pulse profile and resulting in a slow gain depletion. The slow gain deple- 
tion decreases self-phase modulation and carrier heating and carrier cooling effects. Avoiding these nonlinearities and the gain competition among wavelength channels makes multiwavelength operation feasible.

${ }^{1}$ J.-P. Blondel, in Proceedings of IEEE 27th European Conference on Optical Communications ECCC'O1 (IEEE, New York, 2001), pp. 50-53

${ }^{2}$ K. Lee and C. Shu, IEEE J. Quantum Electron. 33, 1832 (1997).

${ }^{3}$ H. Shi, J. Finlay, G. A. Alphonse, J. C. Connolly, and P. J. Delfyett, IEEE Photonics Technol. Lett. 9, 1439 (1997).

${ }^{4}$ P. J. Delfyett, C. DePriest, and T. Yilmaz, IEEE Circuits Devices Mag. 18, 28 (2002).

${ }^{5}$ M. Mielke, G. A. Alphonse, and P. J. Delfyett, IEEE Photonics Technol.
Lett. 15, 501 (2003).

${ }^{6}$ K. L. Hall, G. Lenz, A. M. Darwish, and E. P. Ippen, Opt. Commun. 111, 589 (1994)

${ }^{7}$ P. J. Delfyett, L. T. Florez, N. Stoffel, T. Gmitter, N. C. Andeadakis, Y. Silberberg, J. P. Heritage, and G. A. Alphonse, IEEE J. Quantum Electron. 28, 2203 (1992).

${ }^{8}$ G. A. Alphonse, D. B. Gilbert, M. G. Harvey, and M. Ettenberg, IEEE J. Quantum Electron. 24, 2454 (1988).

${ }^{9}$ Y. Silberberg, P. W. Smith, D. J. Eilenberger, D. A. B. Miller, A. C. Gossard, and W. Wiegmann, Opt. Lett. 9, 507 (1984).

${ }^{10}$ E. B. Treacy, IEEE J. Quantum Electron. 5, 454 (1969).

${ }^{11}$ L. A. Jiang, S. T. Wong, M. E. Grein, E. P. Ippen, and H. A. Hauss, IEEE J. Quantum Electron. 38, 1047 (2002). 